\title{
An Experimental Study of Sub-1 GHz Frequency-Hopping-Based 6LoWPAN Mesh Networking for Smart-Grid Applications
}

\author{
Wonsoo Kim (D), ${ }^{1}$ Kumaran Vijayasankar $\mathbb{D}^{2},{ }^{2}$ Mickael Chouteau $\mathbb{D D}^{3},{ }^{3}$ and Jianwei Zhou (iD ${ }^{4}$ \\ ${ }^{1}$ System Engineering and Marketing, Industrial Systems, Grid Infrastructure, Texas Instruments, Dallas, TX 75243, USA \\ ${ }^{2}$ Embedded Processor, Connected MCU, Low Power RF, Texas Instruments, Dallas, TX 75243, USA \\ ${ }^{3}$ Industrial Accounts Support, Texas Instruments France, Issy-les-Moulineaux 92130, France \\ ${ }^{4}$ Kilby Labs, Texas Instruments, Dallas, TX 75243, USA \\ Correspondence should be addressed to Wonsoo Kim; wonsoo.kim@ti.com
}

Received 12 October 2018; Revised 28 January 2019; Accepted 10 April 2019; Published 16 May 2019

Guest Editor: Amir Rastegarnia

Copyright ( $\odot 2019$ Wonsoo Kim et al. This is an open access article distributed under the Creative Commons Attribution License, which permits unrestricted use, distribution, and reproduction in any medium, provided the original work is properly cited.

\begin{abstract}
This paper presents an experimental study of a multihop Internet Protocol Version 6 over Low-Power Wireless Personal Area Networks (6LoWPAN)-based mesh system that uses un-slotted channel hopping (USCH) medium access control (MAC). Designers of wireless smart-grid networks are using (or looking to use) the Sub-1 GHz spectrum, given its longer radio range compared to the traditional $2.4 \mathrm{GHz}$ spectrum used in $\mathrm{Wi}$ - $\mathrm{Fi}^{\circledR}{ }^{\circledR}$ or Bluetooth ${ }^{\circledR}$ low energy. The frequency-hopping $(\mathrm{FH})$ technique is attractive in this area, as it provides improved robustness and longer range owing to the higher transmission power that regulatory requirements allow. Although Sub-1 GHz allows for a longer range, smart-grid networks deployed over a large geographic area still require multiple hops to provide the required coverage. Many proprietary and standards-based solutions have been proposed to implement such a networking protocol. Notable specifications in this area include the Field Area Networks (FAN) specification from the Wi-SUN (smart utility networks) Alliance and the JupiterMesh specification from the Zigbee Alliance. Little is known about the performance of FH systems over a multihop network, however. This paper presents an implementation of an FH-based multihop networking protocol based on the Texas Instruments (TI) SimpleLink ${ }^{\mathrm{TM}}$ TI 15.4-stack, with an experimental study of such a system over multiple hops compared to a non-frequency-hopping (non-FH) system. Results show that the proposed FH-based 6LoWPAN mesh system significantly improves network coverage, network capacity, and communication robustness to interference while demonstrating coexistence capabilities.
\end{abstract}

\section{Introduction}

Smart-grid networks are used in applications such as automatic meter reading (AMR) and advanced metering infrastructure (AMI). This gives utility providers control over their network, enabling improved billing, network maintenance, and other utility services. Smart-grid networks are typically deployed as multiple subnetworks, each covering a large area like a city or town. Even a single subnetwork is expected to cover a large geographical area.

A single gateway (also known as root node or coordinator) maintains end devices and provides backhaul connectivity to the central network management system. Gateways are generally more expensive to install and maintain compared to multiple end devices (meters are typically located in residential areas). Hence, a single gateway is expected to cover many end devices.

The current challenges in smart-grid networks include

(i) Range: Network deployments can encounter scenarios where houses are far apart, especially in rural areas, requiring a single link to span more than $500 \mathrm{~m}$ to $1 \mathrm{~km}$. This requires a radio technology that can help achieve such long distances. The Sub$1 \mathrm{GHz}$ radio is in general better suited for such requirements. In regions like the United States, the FCC regulation allows the use of a higher transmission power when using FH systems, further 
increasing the achievable range to be in the order of thousands of meters [1]. Even in network scenarios where the houses may be close to each other, like in urban areas, a longer range would help achieve a lower average number of hops, improving overall network performance.

(ii) Coverage: Larger network coverage area requirements generally require multiple hops to reach all end devices. Mesh networking addresses the connectivity issue through multihop transmissions when the data collector and smart meters are not reachable to each other.

(iii) Scale: A large network area also implies that the same network is serving a larger number of end devices. Typical smart-grid networks might cover thousands of homes and thus require a protocol that can scale with such a large network size.

(iv) Interference: The Sub- $1 \mathrm{GHz}$ spectrum is an unlicensed band, and other network operations are possible. According to [2], radio technologies such as wireless sensor networks, telemetry networks, cordless telephones, and mobile phones over the European Global System for Mobile Communications band can cause significant in- or out-of-band noises on the $868 \mathrm{MHz}$ and $915 \mathrm{MHz}$ frequency bands. Given the large network deployment area, different parts of the network could face interference from different networks, and traditional methods like moving to a different channel of operation may not always be feasible.

(v) Coexistence: Coexisting multiple networks deployed by multiple service providers in the same region will be common in unlicensed radio bands. Even with a sole service provider, it may be necessary to maintain multiple overlapping networks in some regions of the deployment from a network management perspective. Such cases require a network that can be robust against traffic from other networks.

An FH-based wireless mesh networking protocol operating in the Sub- $1 \mathrm{GHz}$ spectrum has the potential to address all of these challenges. $\mathrm{FH}$ is a technique of transmitting data by switching one of many channels, where a pseudo-random sequence known to both sender and receiver selects the channel. This technique is known to be robust against interference, with excellent coexistence performance [3-5].

Many proprietary and standards-based solutions have been proposed to implement such a networking protocol. Notable standard specifications in this area include FAN from the Wi-SUN Alliance and JupiterMesh from the Zigbee Alliance. Performance of smart-grid networks using multihop mesh networks has been studied [6]. However, there has been little study on achievable network performance with such systems in the presence of multiple hops and interference.
This paper presents a system design of an FH-based multihop wireless mesh protocol based on the TI 15.4-stack solution implemented over its CC1310, CC1312, or MSP432 ${ }^{\mathrm{TM}}$ devices. The layering architecture is identical to the Wi-SUN FAN specification, but its modular design makes it modifiable to other proprietary or standard specifications. We will also present results based on experiments conducted on the proposed system design with different network conditions.

\section{System Overview}

The FH-based 6LoWPAN mesh network stacks are implemented in software. The software stacks can run in multiple TI microcontroller units (MCUs) such as MSP432, CC1310, and CC1312 wireless MCUs [7-9] or TI microprocessor units (MPUs) such as AM3358 [10]. Regardless of device choice, the software architecture is identical; thus, the devices are interoperable with each other.

The software is available in the form of TI reference designs [11-14]. Figure 1 shows the overall system architecture, which is based on the TI 15.4-stack solution. The TI SimpleLink physical layer (PHY) supports Institute for Electrical and Electronics Engineers (IEEE) 802.15.4 g for 200 and $50 \mathrm{kbps}$ frequency-shift keying and $5 \mathrm{kbps}$ longrange mode. The Sub- $1 \mathrm{GHz}$ radio frequencies on the CC1310 and CC1312 MCUs can support the 902, 863, and $433 \mathrm{MHz}$ frequency bands.

2.1. SimpleLink TI 15.4 Protocol. The TI 15.4-stack supports IEEE 802.15.4-based beacon and nonbeacon modes of operation and a USCH-based Wi-SUN FAN specification [15]. In the USCH mode of operation, the total frequency range is divided into multiple subchannels of a specific receiver bandwidth. Upon device start, each node will start with a random slot number and then tune its receiver to a specific frequency (determined as a function of slot number and device address) and continue to receive on that channel for the channel dwell time (CDT). At the end of CDT, the device will increment the slot number and tune its receiver to the next channel, determined by the slot number and device address.

Each device will hop into different channels based on its device address and the time at which it started. No two devices are synchronized with each other for unicast channel hopping. The gateway can start its own "broadcast hopping sequence" apart from the unicast hopping sequence. The broadcast hopping sequence consists of a broadcast interval and a broadcast dwell time. A representation of network's broadcast hopping sequence and device's unicast hopping sequence is shown in Figures 2 and 3, respectively.

All devices in the network will

(i) Follow the controller's broadcast hopping sequence

(ii) Follow their unicast hopping sequence during the broadcast dwell interval and will switch to a broadcast channel during the broadcast dwell time 


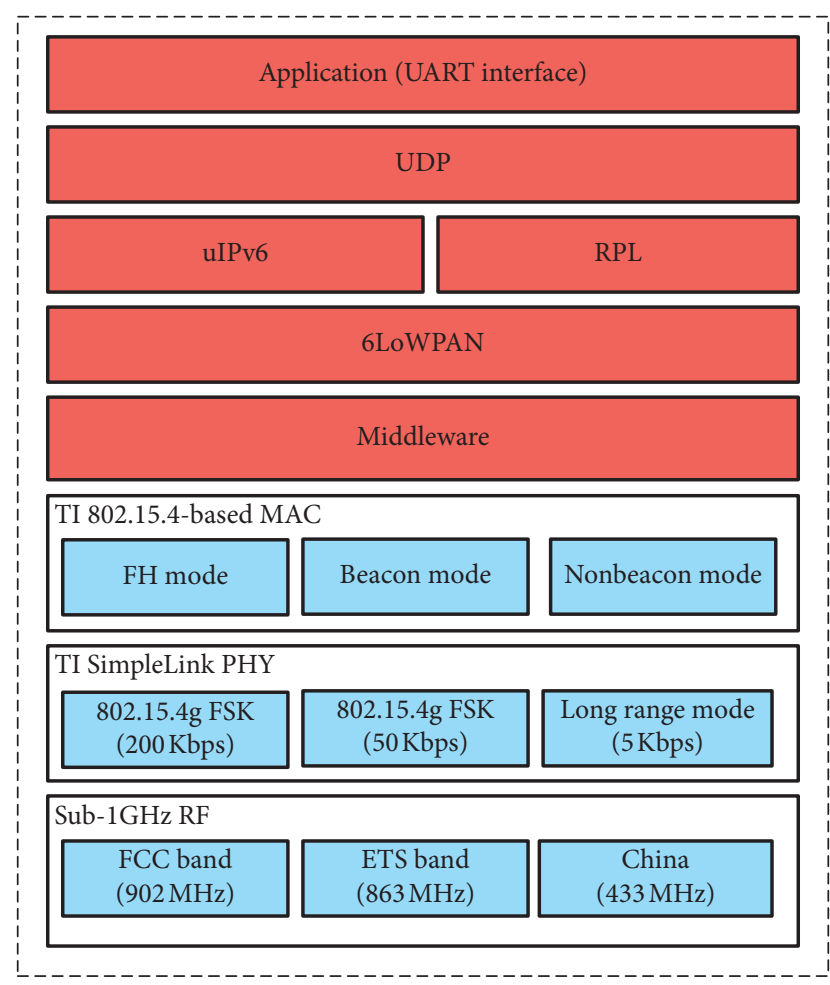

Figure 1: FH-based 6LoWPAN mesh system architecture.

(iii) Propagate the broadcast hopping sequence and its unicast hopping sequence through asynchronous transmissions based on trickle timers, as defined in [15]

The initial device discovery and channel hopping exchange occurs using asynchronous transmissions (where a discovery packet is sent on all channels back to back), as explained in [15]. During steady state, a transmitter device will first determine the channel on which a receiver is currently listening and then direct its transmission onto that specific channel, as explained in Figure 4. This is called receiver-directed transmissions.

Every data frame will carry with its timing information an information element format that the receiver will use to update a neighbor table. Using the information in the neighbor table, a device can track all of its neighbors in terms of the channel in which they will be listening. The Wi-SUN FAN specification offers more details about device discovery, channel hopping synchronization, and timing estimation [15].

The experimental study compares the USCH mode of operation (representing $\mathrm{FH}$ ) against the performance of the system when the 15.4 MAC is in the nonbeacon (non-FH) mode of operation.

2.2. 6LoWPAN Adaptation Protocol. The goal of the 6LoWPAN protocol is to support IP services by reducing the gap between IPv6 and lower stacks, to serve IPv6 applications on low-end devices typically restricted in terms of processing power, memory, and energy. The primary tasks of
6LoWPAN are fragmentation and reassembly, IPv6 and User Datagram Protocol (UDP) header compression, stateless IPv6 address autoconfiguration, and neighbor discovery optimization $[16,17]$.

2.3. RPL Routing Protocol. The 6LoWPAN mesh system presented in this paper uses the IPv6 Routing Protocol for Low-Power and Lossy Networks (RPL) for multihop routing $[18,19]$. Network formation with RPL routing is initiated by broadcasting a destination-oriented directed acyclic graph (DODAG) information object (DIO) by the root node. Once child nodes receive the DIOs, they broadcast the DIOs and send back a unicast destination advertisement object (DAO) packet to the parents with the best route toward the root. A trickle algorithm determines DIO and DAO transmission times [20]. The RPL routing metric to decide the best route can include multiple choices. In our experiments, the RPL routing protocol uses the expected transmission count by default.

\section{Experimental Setup}

The experimental study uses two types of UDP applications: UDP poll and UDP push. In the UDP poll application, the gateway initiates an application-level read-request message to each node to read data. The end devices respond with a UDP read-response message of the same size. In the UDP push application, each node initiates data transmissions whenever there is application data to send.

These two UDP applications represent the traffic profile of typical AMI applications. The Device Language Message Specification (DLMS) and Companion Specification for Energy Metering (COSEM) for smart metering applications use the UDP poll-based mechanism [21], and the Constraint Application Protocol (CoAP) uses a mix of UDP poll (GET command) and push (periodic OBSERVE command) mechanisms [22].

All the experiments were performed over real channel with $902 \mathrm{MHz}$ ISM frequency bands, not based on simulated channel models. To validate interference impact on the network performance, TI SmartRF ${ }^{\mathrm{TM}}$ Studio [23] was used to generate interferences to the network. Figure 5 shows the noise source characteristic captured by a spectrum analyser, showing that the noise source jammed eight channels, with channel space of $200 \mathrm{KHz}$, based on reference threshold of $-10 \mathrm{dBm}$.

Table 1 summarizes the system parameters used in the experiments.

\section{Experimental Results}

The experimental study created a multihop topology with address filtering in the software. A node can listen to all incoming packets from the neighbors but accept packets coming from a set of nodes configured in the software at lower layers. Although such an approach avoids spatial reuse over multihop networks, it helps study the performance of multihop networks, with an expected topology 


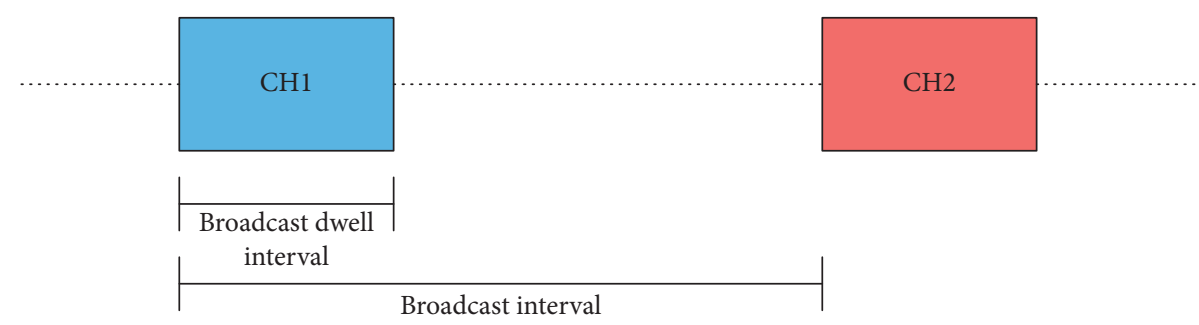

FIgURE 2: An example broadcast FH sequence.

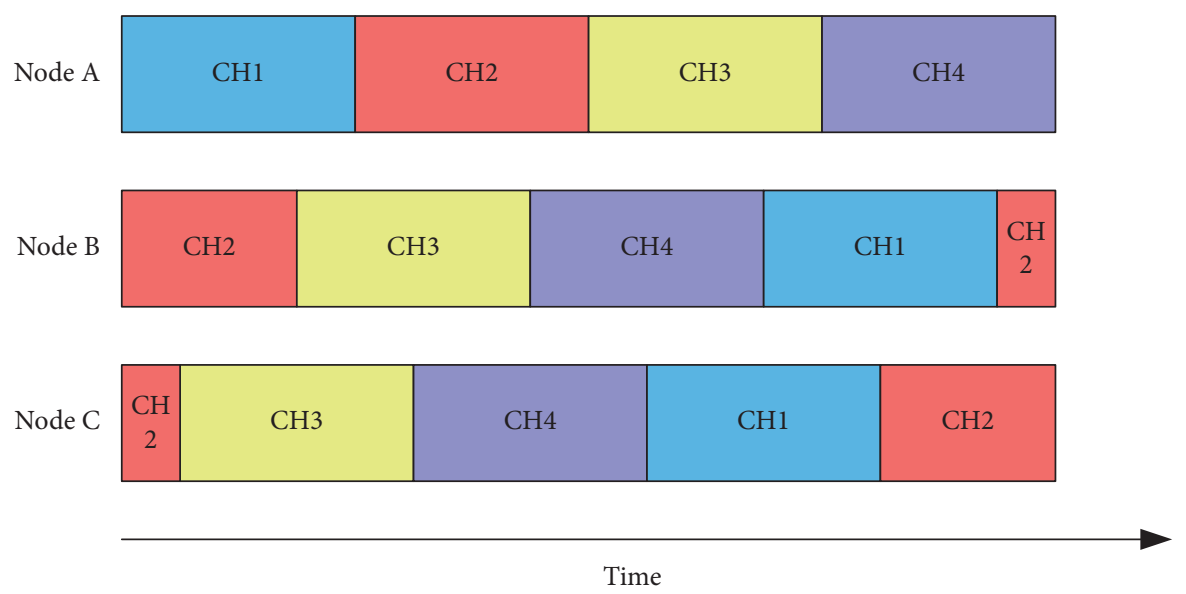

Figure 3: An example unicast FH sequence.

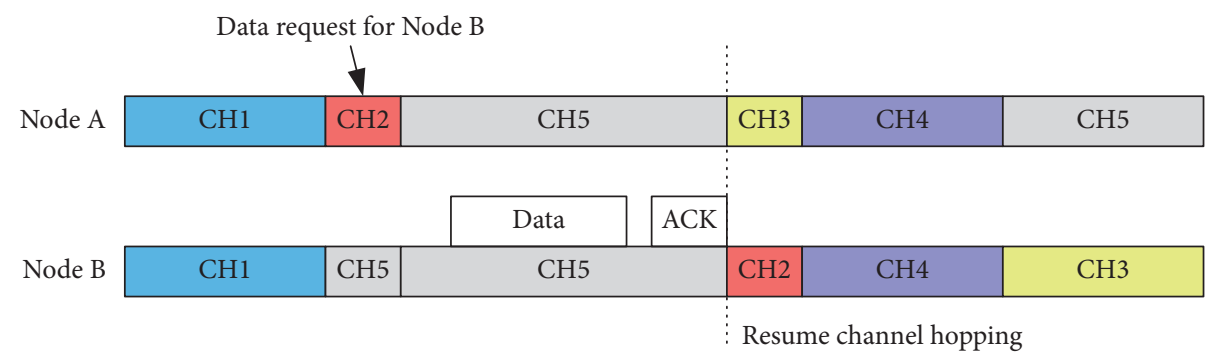

FIgURE 4: Receiver-directed transmissions.

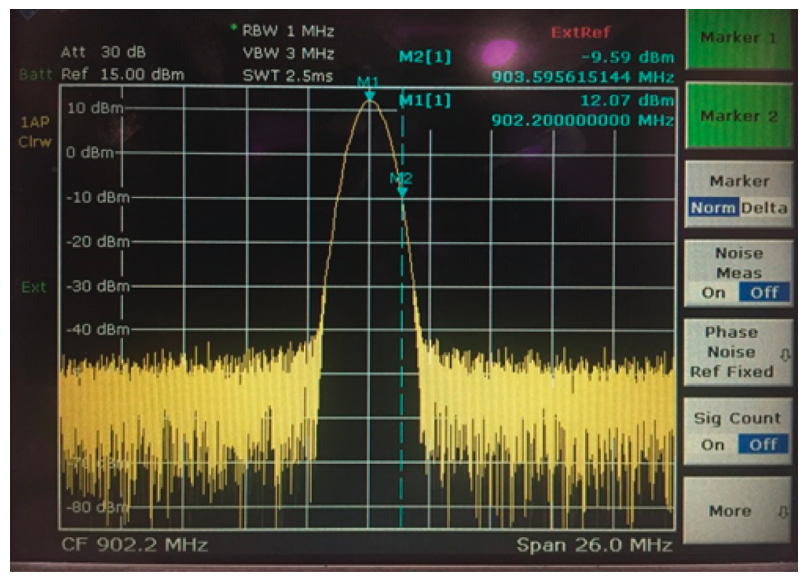

Figure 5: Noise source with TX power of $14 \mathrm{dBm}$.
TABLE 1: System parameters.

\begin{tabular}{lc}
\hline Parameter & Value \\
\hline Channel bandwidth & $200 \mathrm{kHz}$ \\
Total number of channels & 129 \\
Unicast dwell time & $250 \mathrm{msec}$ \\
PHY data rate & $50 \mathrm{kbps}$ \\
\hline
\end{tabular}

enabling a better understanding of network performance results.

We performed five different experiments to study the impact of

(i) Multiple hops and continuous external interference

(ii) Packet sizes and intermittent external interference 
(iii) In-band interference

(iv) Coexistence of multiple networks

(v) Large network size

4.1. Impact of Multihop and External Interference on System Performance. To study the impact of multiple hops, see the six-hop linear topology shown in Figures 6 and 7. The UDP poll application generates a read request with a packet size of $100 \mathrm{~B}$ every $5 \mathrm{~s}$.

Software filtering in receive path allows creating multihop networks even with nodes located within transmission ranges. The external noise source, shown in Figure 5, was generated over real channel to verify the impact of interference on system performance.

We measured these performance metrics:

(i) The end-to-end delivery ratio: The ratio of number of successful read responses to the number of read requests measured at the gateway. The read was considered a failure if the packet read request failed to reach the end device, or if the read response from the end device failed to reach the gateway. Figure 8 presents the experimental results. The average delivery ratio over six-hop networks was 99.7 percent, and the worst-case performance was 99.4 percent at a six-hop distance.

(ii) Round-trip time (RTT): The time taken to perform a successful read measure, from the time a gateway initiates a read request to the time it took to receive the corresponding read response. Figure 9 presents RTT performance with and without noise. As expected, RTT increases as the number of hops increases. This is because more hops increase the number of transmissions to reach at the destination. Comparing the RTT performance with and without noise, the RTT gap increases as the number of hop increases. This increase is due to the nature of the TI 15.4-stack, where the delay is a trade-off to achieve improved reliability. Also, the RTT for devices at farther hops is higher, owing to the fact that the delay accumulates for devices that are multiple hops away. The results show that, for the one-hop distance, the average RTT performance is $0.19 \mathrm{~s}$ regardless of noise. For the six-hop distance, the RTT performance is $1.17 \mathrm{~s}$ and $1.38 \mathrm{~s}$ for the $\mathrm{FH}$ system without and with noise, respectively.

(iii) Goodput: Figure 10 presents the achievable throughput based on the RTT accounting for the network overheads from different protocol layers. The results show that the goodput performance varies from $8.3 \mathrm{kbps}(100 \mathrm{~B}$ at the one-hop distance) to $1.2 \mathrm{kbps}$ (100 B at the six-hop distance).

The results show that the system scales with the number of hops, providing greater than 99.4 percent reliability with up to six hops. The system is robust even in the presence of external interference. It should be noted that, considering an achievable range of communication in the order of several

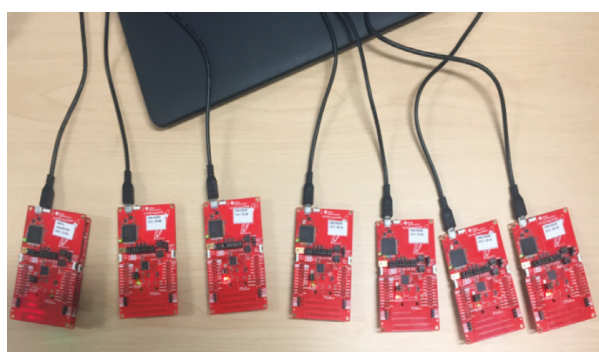

Figure 6: Physical view of the six-hop topology.

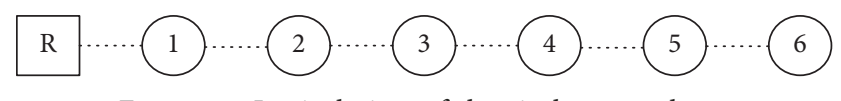

FIgURE 7: Logical view of the six-hop topology.

$100 \mathrm{~m}$ per radio link [1], a six-hop network represents coverage in the order of multiple $\mathrm{km}$ radius, making it suitable for smart-grid networks. The results for a non-FH system was not provided as a constant noise source at the channel of operation would kill a network with non-FH systems.

4.2. Impact of Packet Sizes and Intermittent External Interference on System Performance. The previous experiment applies a constant interference that may not be realistic. In this experiment, we apply a noise source at intermittent levels, with different levels of duty-cycle periods to emulate a more realistic noise source. For comparison, we include the performance results of a non-FH system. In these experiments, $\mathrm{FH}-129$ represents an FH system that is hopping over 129 channels; the non-FH system is operating in nonbeacon mode.

This experiment uses FH-129 and non-FH systems with three different packet sizes: 100, 300, and 500 B. Figure 11 presents the end-to-end delivery ratio. The results show that, for all sizes, the FH-129 system shows no degradation with the noise duty cycle, while the non-FH system shows significant degradation as the noise duty cycle increases. The performance degradation of the non-FH system becomes significant as the data size increases. This is because a bigger data size means a longer transmission time, which increases the chances of errors in noisy channels. For a noise duty cycle of 16 percent, the performance gap between the FH129 and non-FH systems is 33 percent for $100 \mathrm{~B}$ and increases to 78 percent for $500 \mathrm{~B}$.

4.3. Impact of In-Band Interference on System Performance. The goal of this experiment is to verify how reliably the FH129 system performs with in-band interference (mostly packet collisions among nodes) compared to the non-FH system. To verify this, we created a multihop network with two 3-hop branches, as shown in Figure 12. This experiment uses the UDP push application, which is more likely to have packet collisions due to the distributed packet transmissions among end nodes. The application data size is fixed at $500 \mathrm{~B}$.

Figure 13 shows the delivery ratio performance as a function of packet interval. The results show that, compared 


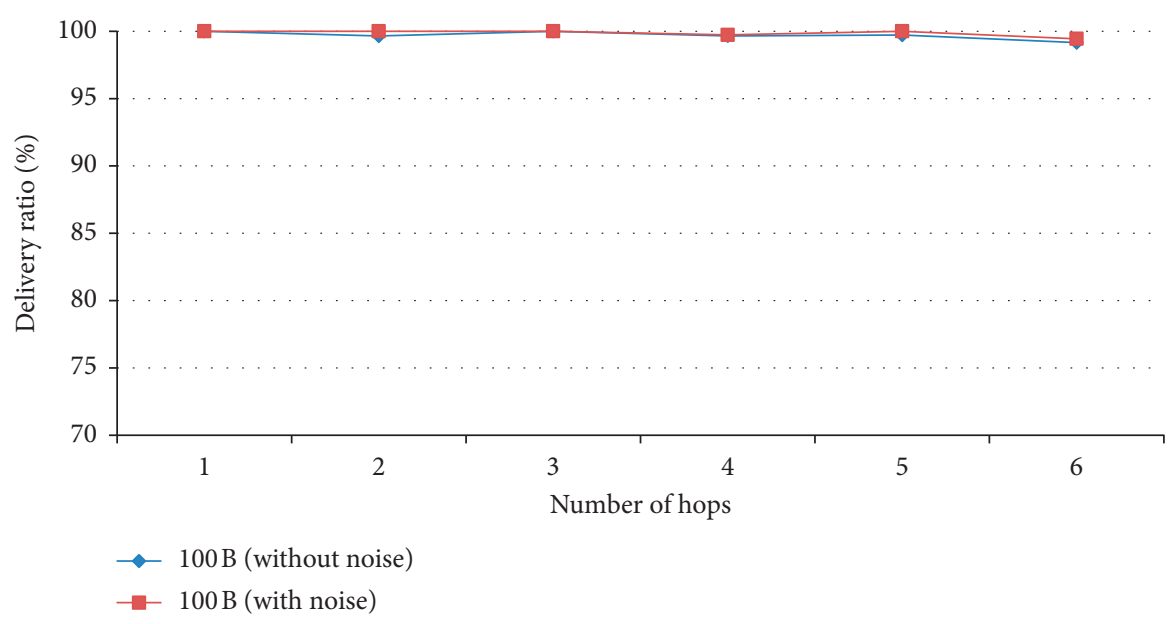

FIgURE 8: Delivery ratio as a function of the number of hops.

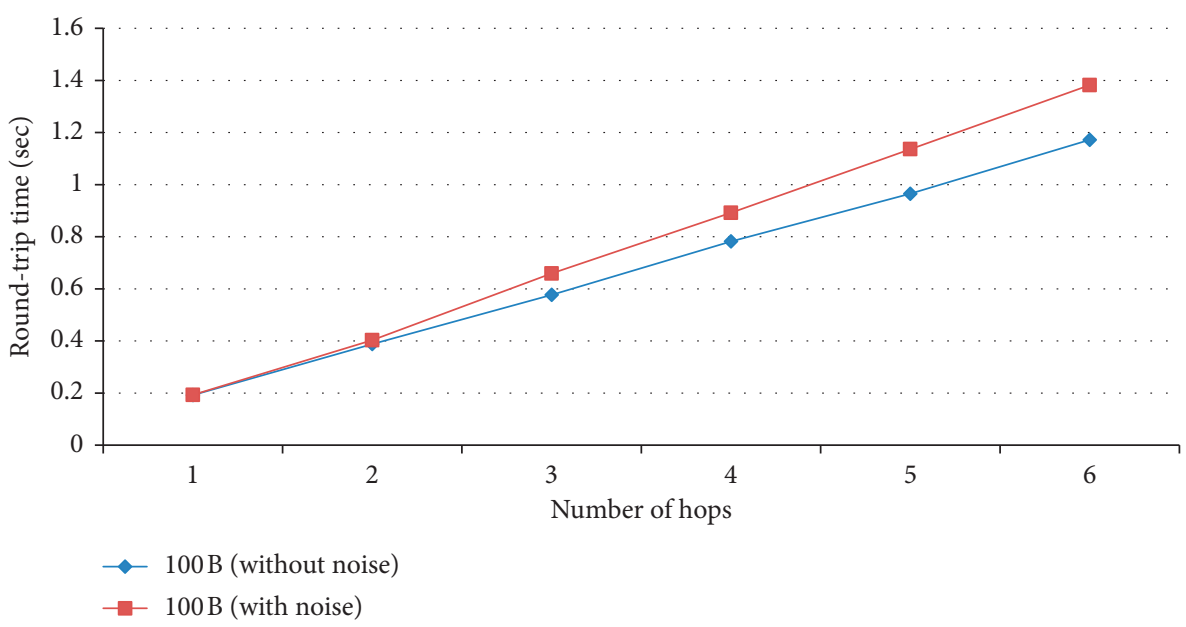

FIGURE 9: RTT as a function of the number of hops.

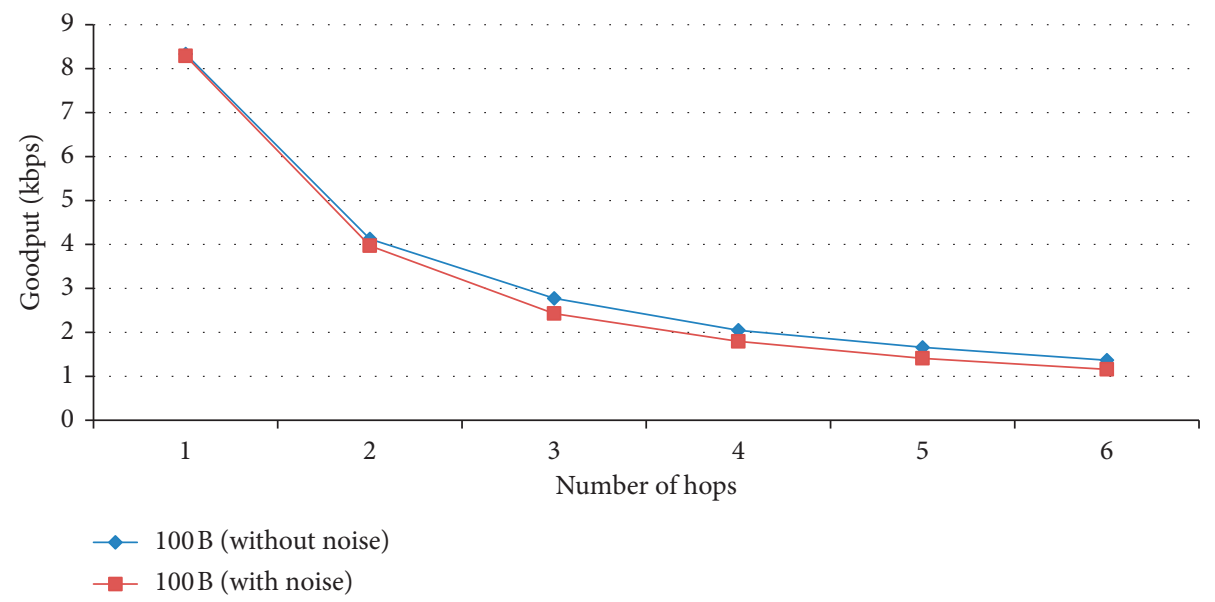

FIGURE 10: Goodput performance as a function of the number of hops.

to the FH-129 system, non-FH system performance has more of an impact on network traffic, since the performance degradation becomes significant as the packet interval decreases. The performance gap between the FH-129 and non-FH systems increases to 29 percent at a 5 s packet interval. This is because the $\mathrm{FH}$ technique in the FH-129 


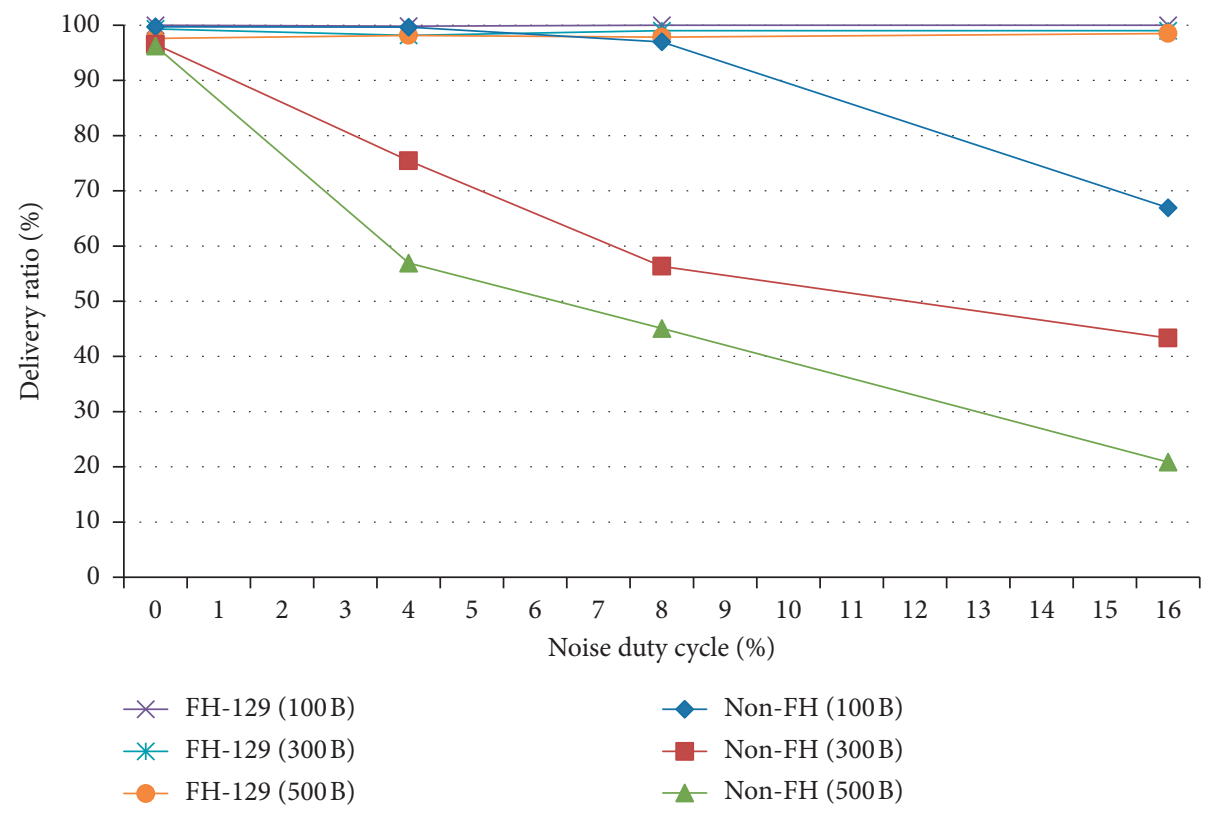

Figure 11: Delivery ratio as a function of noise duty cycle.

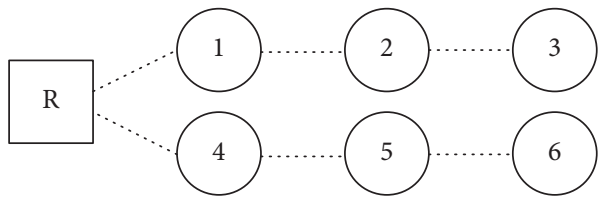

FIGURE 12: Multihop topology with two 3-hop network branches.

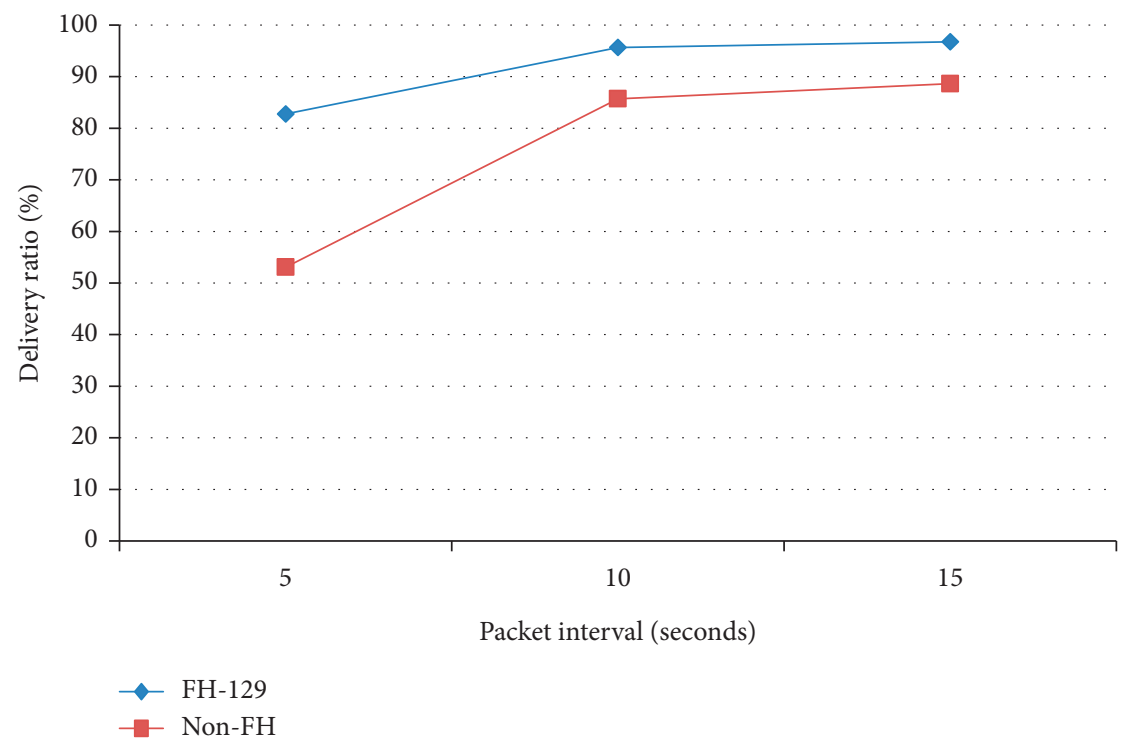

Figure 13: Average delivery ratio (FH-129 vs. non-FH systems).

system significantly reduces collisions among nodes in different branches. For the non-FH system, packet losses occur when there are simultaneous transmissions in more than one node. The impact becomes significant with a higher traffic ratio.
4.4. Impact of Coexistence on System Performance. This experiment studies the impact of another network on the performance of a given network, using a three-hop network with a UDP poll application that generates $500 \mathrm{~B}$ read requests and a $5 \mathrm{~s}$ read interval. 


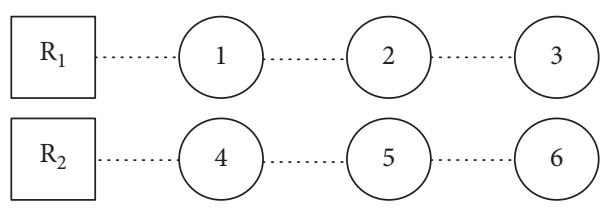

FIGURE 14: Two three-hop networks for the coexistence scenario.

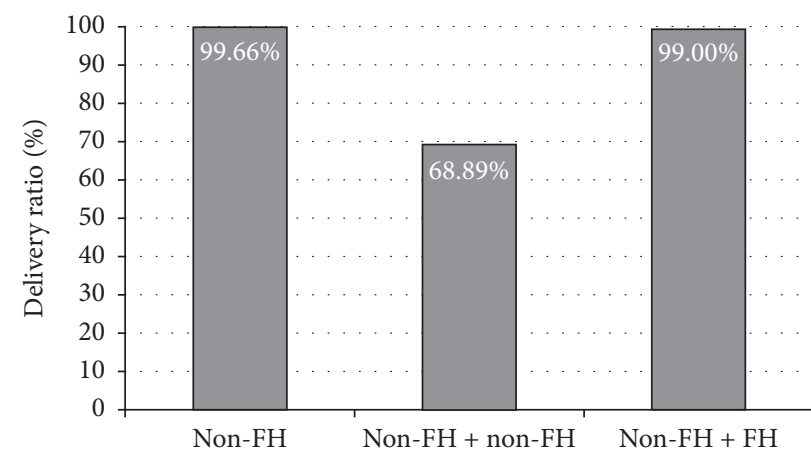

Figure 15: Average delivery ratio (non-FH vs. FH-129 systems)

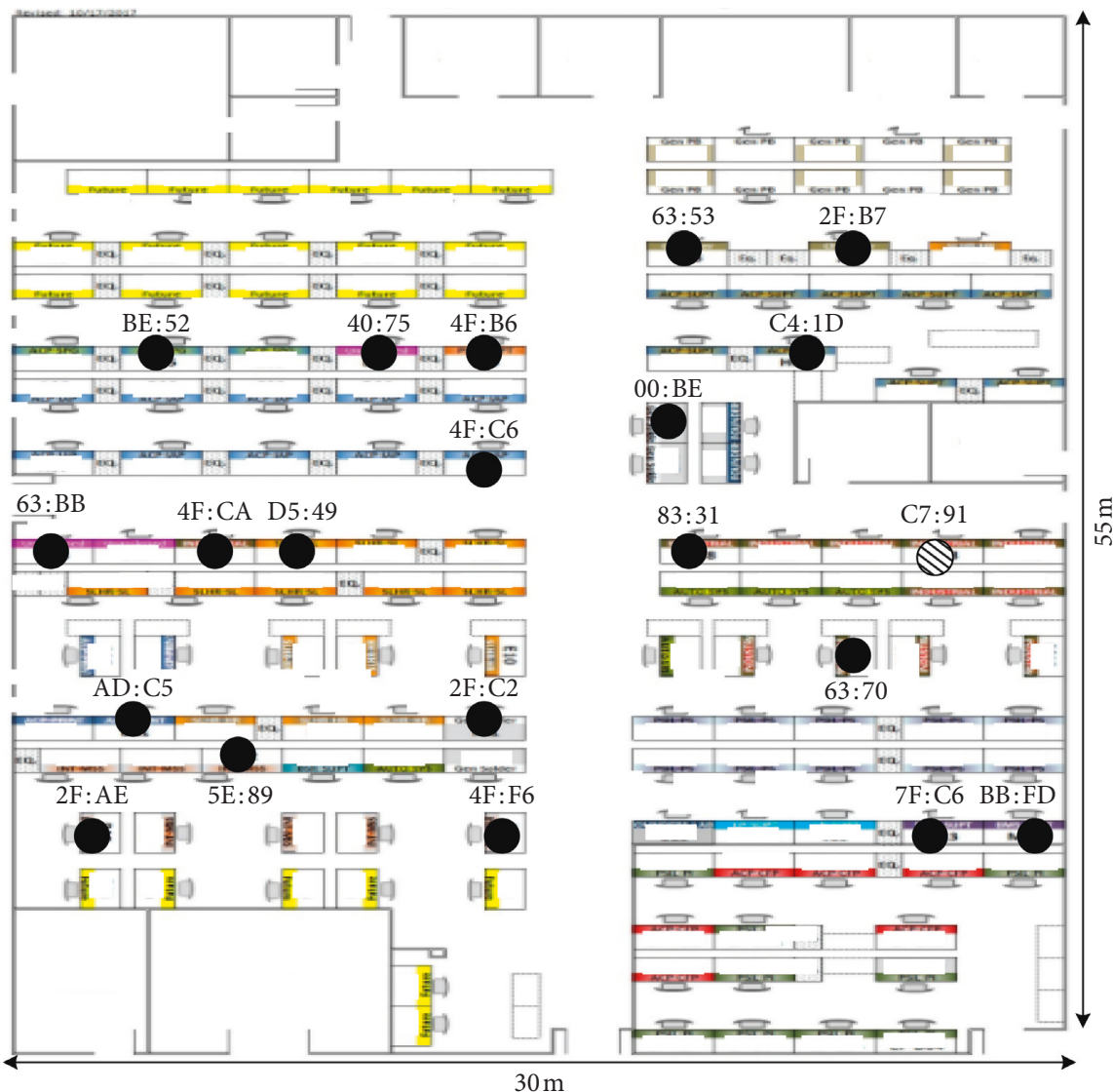

Data collector

End node

FigURE 16: 20-node network setup. 


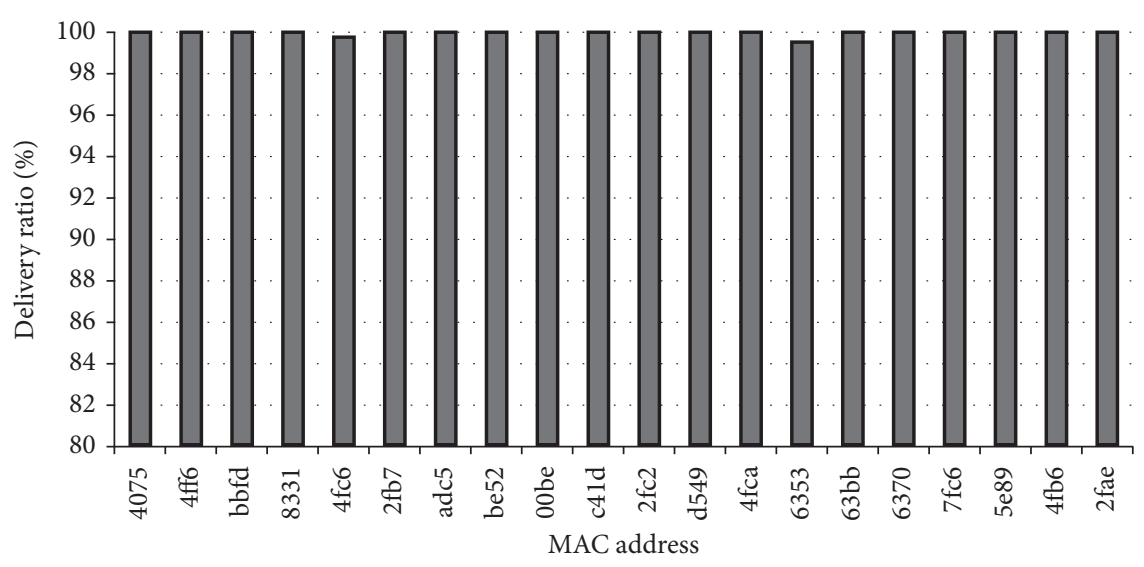

FIGURE 17: Delivery ratio performance with 20-node setup.

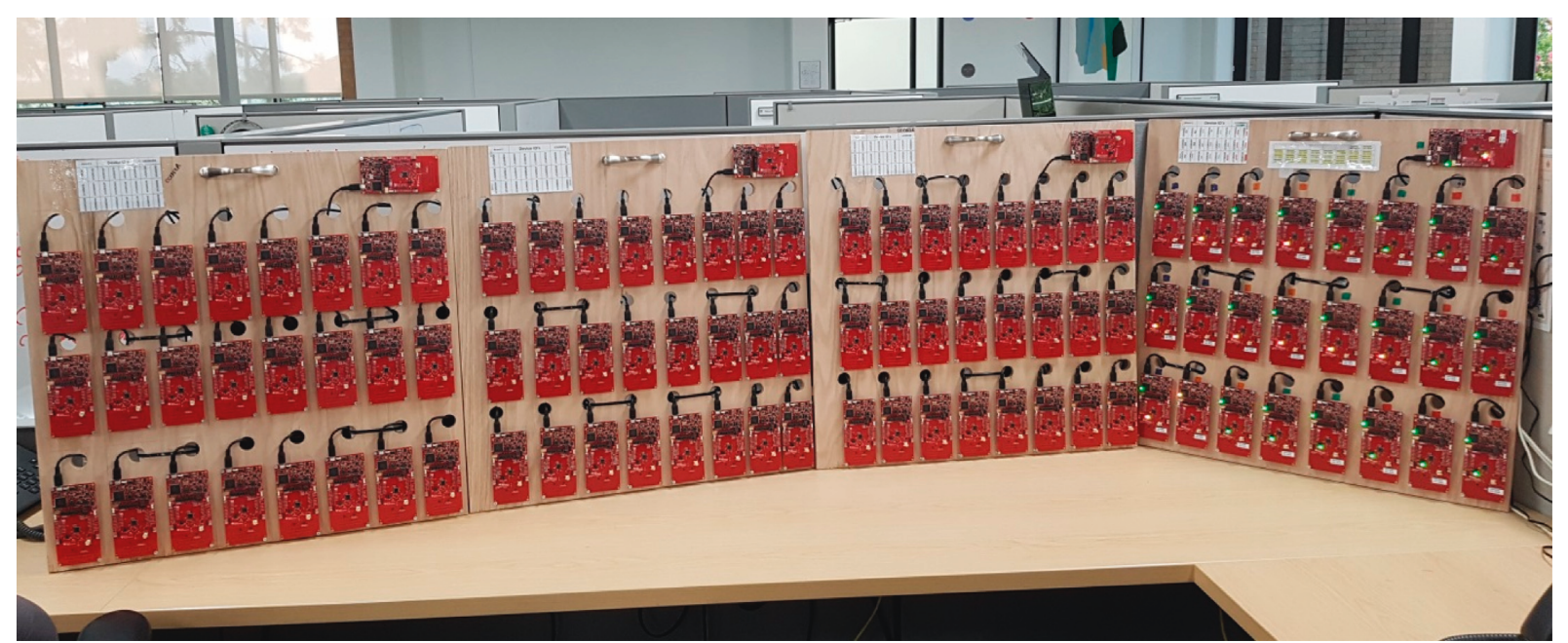

FIGURE 18: 100-node network setup.

We consider three cases:

(i) A single non-FH network operating over three hops

(ii) Two non-FH networks operating in parallel, as shown in Figure 14

(iii) One non-FH and another FH network operating in parallel, also shown in Figure 14

Figure 15 presents the achieved end-to-end delivery ratio. The results show that, for the sole three-hop network of the non-FH system, the delivery ratio is 99.66 percent, while in the coexistence scenario with two nonFH systems, the delivery ratio decreases to 70.23 percent due to adjacent non-FH network interference. However, in the coexistence scenario with FH-129 and non-FH systems, the FH-129 system has no impact on the delivery ratio of the coexisting non-FH system, which proves that the FH-129 system displays outstanding coexistence performance.

4.5. Impact of Network Size on System Performance. This experiment measures delivery ratio performance in a real environment where 20 end nodes and 1 data collector are distributed in the lab. Figure 16 shows the details of the 20node setup with locations and the last two bytes of MAC address information. This experiment does not use the software filtering to create multihop networks. The experiment was run over multiple days in the presence of interference from other lab experiments.

Figure 17 shows the delivery ratio performance as a function of the MAC address of each end node in the 20node setup. This experiment was performed with a UDP poll application with $100 \mathrm{~B}$, and the experiment runs for two days. The average delivery ratio performance achieves 99.97 percent and the average RTT is 0.331 seconds.

This experiment set up a large network of 100 end nodes and a single root node, as shown in Figure 18. The setup does not use software filtering to create multihop networks, but multiple hops are created from network collisions and the proximity of better routers to end devices.

Next, to validate the performance over a large number of nodes, the results of a 100-node network are presented. Figure 19 shows the delivery ratio performance as a function of the MAC address of each node in the 100-node network setup. This experiment uses the UDP poll application, with $100 \mathrm{~B}$ of data running for more than three days. The $X$-axis 


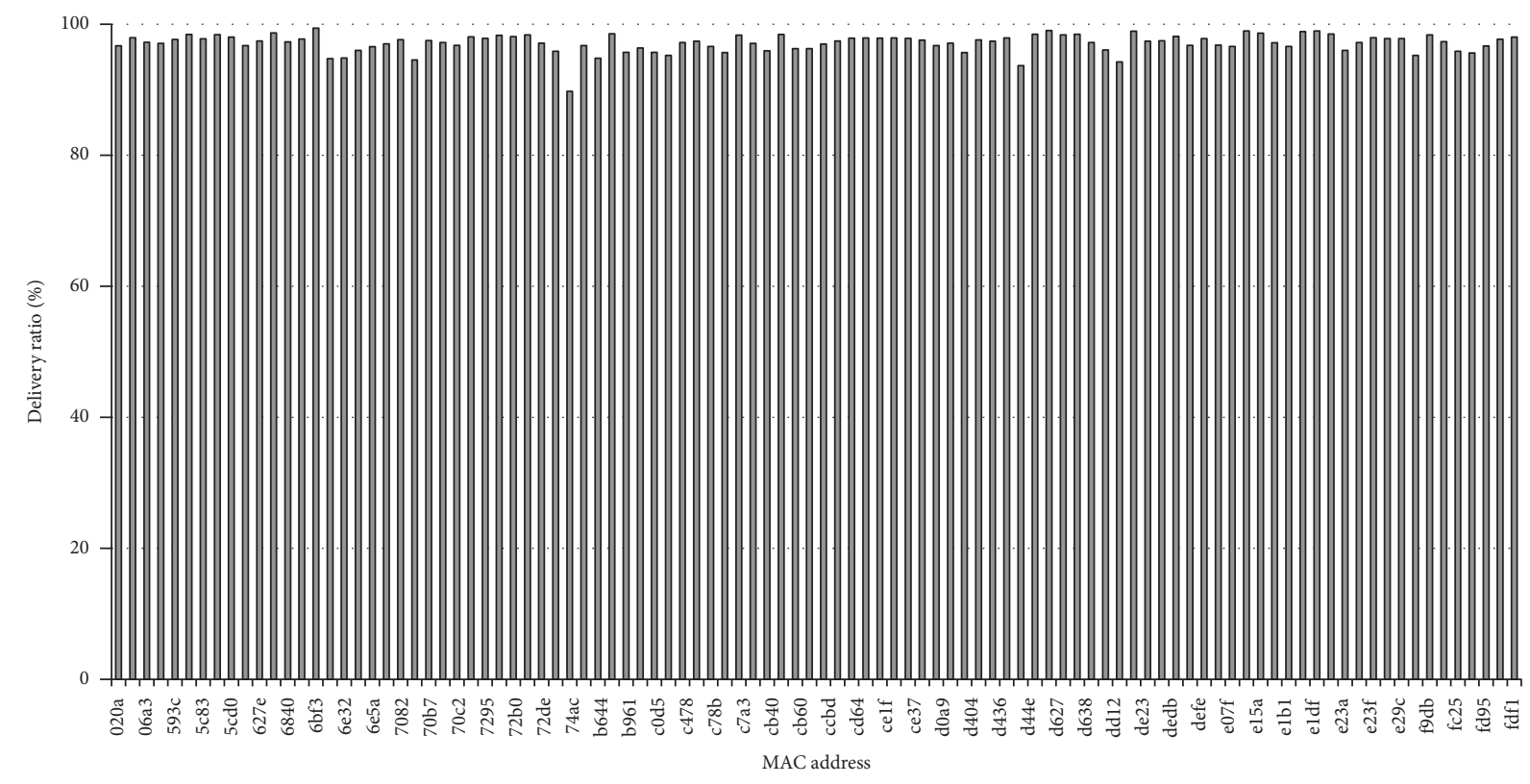

FIGURE 19: Delivery ratio performance with 100-node setup.

shows the MAC address of the end node, and the $Y$-axis shows the average delivery ratio in percentage. The results show that the FH-based mesh system is robust even in the dense network scenario. The average delivery ratio is 97.14 percent.

\section{Conclusion}

The paper presented the challenges in wireless networks for smart-grid applications and the benefits of an FH-based multihop protocol in addressing these challenges, specifically an FH-based multihop network based on the SimpleLink TI 15.4-stack solution over TI CC1310, CC1312, or MSP432 MCUs.

The modular software design can be extended to implement similar wireless smart-grid protocols over supported frequency bands and data rates. The experimental results show that such a system can scale to a large number of devices supporting multiple hops while remaining robust to interference from external sources and other coexisting networks. Considering the range of achievable radio links and the ability of the solution to support multiple hops, network coverage in the order of tens of $\mathrm{km}$ radius can be achieved, making it suitable for large-scale smart-grid networks [1].

\section{Data Availability}

The experimental data used to support the findings of this study are included within the article.

\section{Conflicts of Interest}

The authors declare that there are no conflicts of interest regarding the publication of this paper.

\section{References}

[1] K. Vijayasankar and R. Sandre, Frequency Hopping for LongRange IoT Networks, White Paper, Texas Instruments Inc., Dallas, TX, USA, 2016.

[2] N. Baccour, A. Koubâa, C. Noda et al., Radio Link Quality Estimation in Low-Power Wireless Networks, Springer International Publishing, New York, NY, USA, 2013.

[3] T. Watteyne, A. Mehta, and K. Pister, "Reliability through frequency diversity: why channel hopping makes sense," in Proceedings of the 6th ACM Symposium on Performance Evaluation of Wireless Ad Hoc, Sensor, and Ubiquitous Networks, Tenerife, Spain, October 2009.

[4] V. Navda, A. Bohra, S. Ganguly, and D. Rubenstein, "Using channel hopping to increase 802.11 resilience to jamming attacks," in Proceedings of the IEEE INFOCOM 2007-26th IEEE International Conference on Computer Communications, Anchorage, AK, USA, May 2007.

[5] P. Popovski, H. Yomo, and R. Prasad, "Strategies for adaptive frequency hopping in the unlicensed bands," IEEE Wireless Communications, vol. 13, no. 6, pp. 60-67, 2006.

[6] P. Moulema, W. Yu, G. Xu et al., "On effectiveness of meshbased protocols for smart grid communication networks," ACM SIGAPP Applied Computing Review, vol. 14, no. 2, pp. 59-70, 2014.

[7] Texas Instruments, CC1310: SimpleLink Sub-1 GHz Ultra-Low Power Wireless Microcontroller, Texas Instruments, Dallas, TX, USA, 2015, http://www.ti.com/product/CC1310.

[8] Texas Instruments, CC1312: SimpleLink Sub-1 GHz CC1312R Wireless MCU, Texas Instruments, Dallas, TX, USA, 2018, http://www.ti.com/product/CC1312R.

[9] Texas Instruments, MSP432P401R: SimpleLink Ultra-LowPower 32-Bit ARM Cortex-M4F MCU with Precision ADC, 256KB Flash and 64KB RAM, Texas Instruments, Dallas, TX, USA, 2015, http://www.ti.com/product/MSP432P401R.

[10] Texas Instruments, AM3358: Sitara Processor Arm Cortex-A8, $3 D$ Graphics, PRU-ICSS, Texas Instruments, Dallas, TX, USA, 2011, http://www.ti.com/product/AM3358. 
[11] Texas Instruments, Simple 6LoWPAN Mesh Data Collector Improves Network Performance Reference Design, Texas Instruments, Dallas, TX, USA, 2018, http://www.ti.com/tool/ tida-01547.

[12] Texas Instruments, Simple 6LoWPAN Mesh End-Node Improves Network Performance Reference Design, Texas Instruments, Dallas, TX, USA, 2018, http://www.ti.com/tool/ tida-010003.

[13] Texas Instruments, Secured 6LoWPAN Mesh End-Node with Enhanced Network Capacity Reference Design, Texas Instruments, Dallas, TX, USA, 2018, http://www.ti.com/tool/ tida-010024.

[14] Texas Instruments, Universal Data Concentrator Reference Design Supporting Ethernet, 6LoWPAN RF Mesh and More, Texas Instruments, Dallas, TX, USA, 2018, http://www.ti.com/ tool/tida-010032.

[15] WiSUN Alliance, Technical Profile Specification, Field Area Network, WiSUN Alliance, San Ramon, CA, USA, 2017.

[16] G. Montenegro, N. Kushalnagar, J. Hui, and D. Culler, "Transmission of IPv6 Packets over IEEE 802.15.4 Networks," RFC 4944, 2007, https://www.rfc-editor.org/info/rfc4944.

[17] J. Hui and P. Thubert, "Compression Format for IPv6 Datagrams over IEEE 802.15.4-Based Networks,” RFC 6282, 2011, https://www.rfc-editor.org/info/rfc6282.

[18] T. Winter, P. Thubert, A. Brandt et al., "RPL: IPv6 Routing Protocol for Low-Power and Lossy Networks," RFC 6550, 2012, https://www.rfc-editor.org/info/rfc6550.

[19] H.-S. Kim, J. Ko, D. E. Culler, and J. Paek, "Challenging the IPv6 routing protocol for low-power and lossy networks (RPL): a survey," IEEE Communications Surveys \& Tutorials, vol. 19, no. 4, 2017.

[20] P. Levis, T. Clausen, J. Hui, O. Gnawali, and J. Ko, "The Trickle Algorithm," RFC 6206, 2011, https://www.rfc-editor.org/info/ rfc6206.

[21] International Electrotechnical Commission, "Electricity metering data exchange - the DLMS/COSEM suite-part 5-3: DLMS/COSEM application layer," IEC 62056-5-3:2017, IEC, Geneva, Switzerland, 2017.

[22] Z. Shelby, K. Hartke, and C. Bormann, "The Constrained Application Protocol (CoAP)," RFC 7252, 2014, https://www. rfc-editor.org/info/rfc7252.

[23] Texas Instruments, SmartRF Studio, Texas Instruments, Dallas, TX, USA, 2010, http://www.ti.com/tool/smartrftmstudio. 


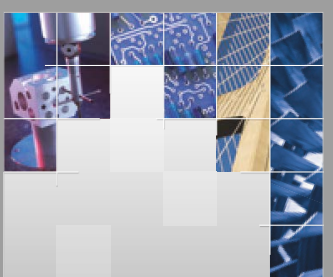

\section{Enfincering}
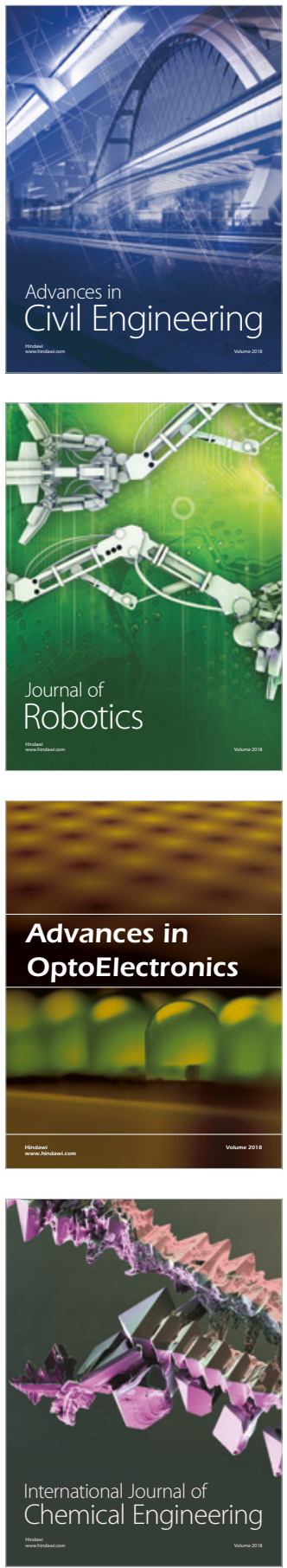

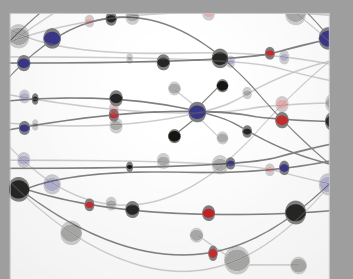

\section{Rotating \\ Machinery}

The Scientific World Journal

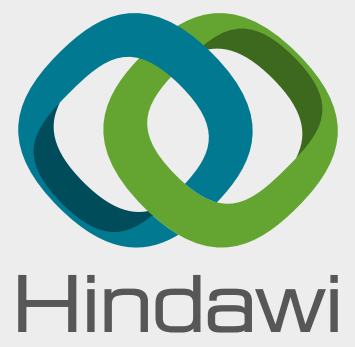

Submit your manuscripts at

www.hindawi.com
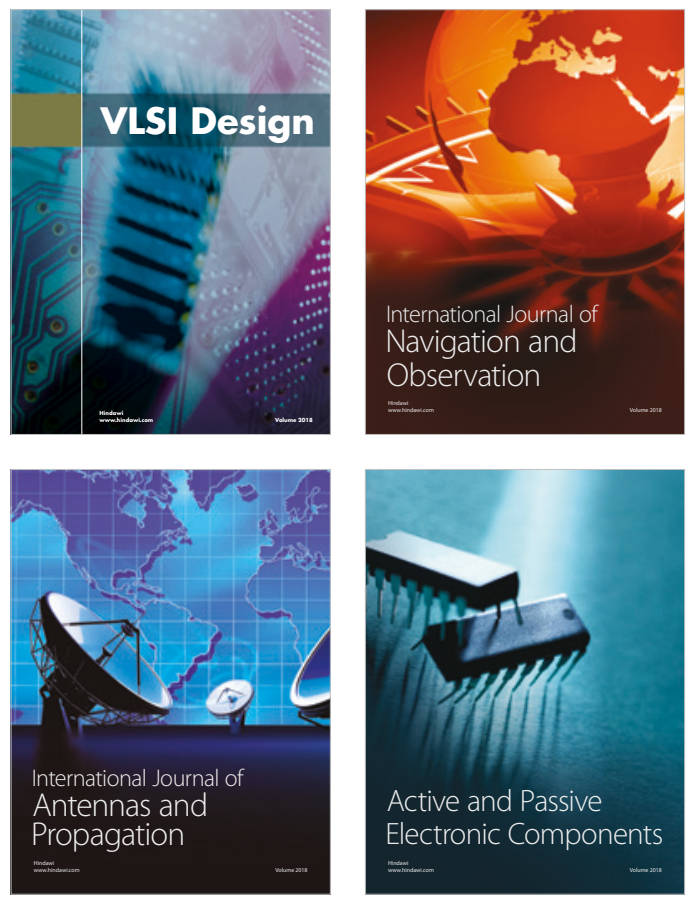
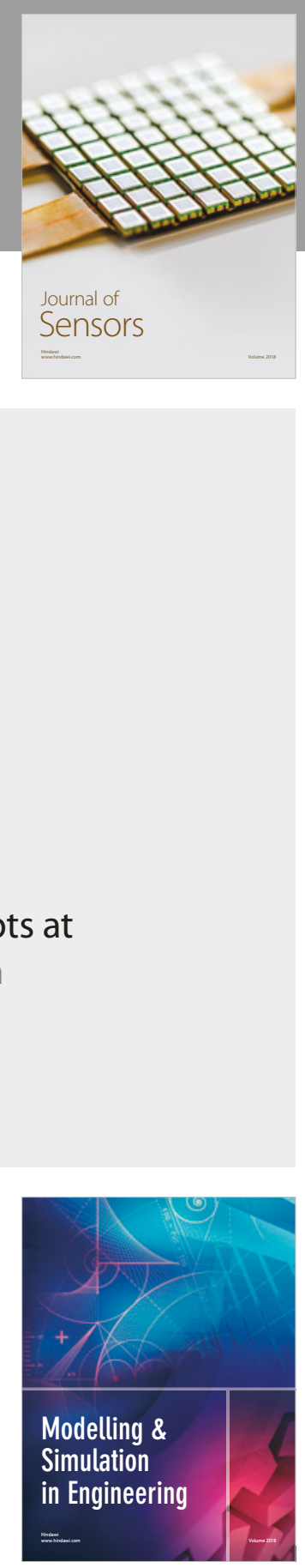

\section{Advances \\ Multimedia}
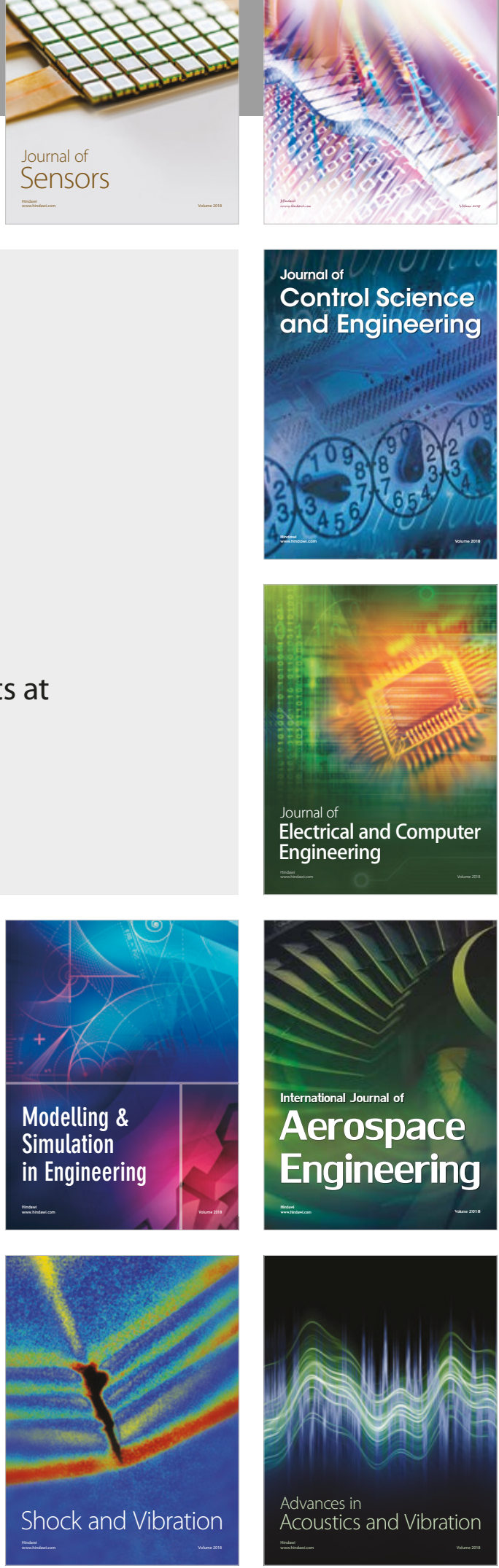